\title{
Estimation of Regularization Parameters in Elliptic Optimal Control Problems by POD Model Reduction
}

\author{
Martin Kahlbacher and Stefan Volkwein \\ Institute for Mathematics and Scientific Computing, \\ University of Graz, Heinrichstrasse 36, 8010 Graz, Austria \\ martin.kahlbacher@uni-graz.at, stefan.volkwein@uni-graz.at
}

\begin{abstract}
In this article parameter estimation problems for a nonlinear elliptic problem are considered. Using Tikhonov regularization techniques the identification problems are formulated in terms of optimal control problems which are solved numerically by an augmented Lagrangian method combined with a globalized sequential quadratic programming algorithm. For the discretization of the partial differential equations a Galerkin scheme based on proper orthogonal decomposition (POD) is utilized, which leads to a fast optimization solver. This method is utilized in a bilevel optimization problem to determine the parameters for the Tikhonov regularization. Numerical examples illustrate the efficiency of the proposed approach.
\end{abstract}

\section{Introduction}

Parameter estimation problems for partial differential equations are very important in application areas. Using Tikhonov regularization techniques (see, e.g., [20]) these problems can often be expressed in terms of constrained optimal control problems so that numerical optimization can be applied to solve the parameter identification problems numerically. Here, we apply an augmented Lagrangian method (see, e.g., [23]) combined with a globalized sequential quadratic programming (SQP) algorithm as described in [6]. In this article we continue our successful development of solution methods for parameter estimation problems for nonlinear elliptic partial differential equations (PDEs);

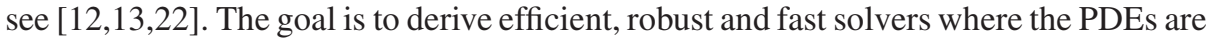
discretized by a Galerkin scheme based on proper orthogonal decomposition. POD is a powerful method to derive low-dimensional models for nonlinear systems. It is based on projecting the system onto subspaces consisting of basis elements that contain characteristics of the expected solution. This is in contrast to, e.g., finite element techniques, where the elements of the subspaces are uncorrelated to the physical properties of the system that they approximate. It is successfully used in different fields including signal analysis and pattern recognition (see, e.g., [5]), fluid dynamics and coherent structures (see, e.g., [7|15]) and more recently in control theory (see, e.g., [10]). The relationship between POD and balancing is considered in [9 1923]. In contrast to POD approximations, reduced-basis element methods for parameter dependent elliptic systems are investigated in [1816]18], for instance.

A. Korytowski et al. (Eds.): System Modeling and Optimization, IFIP AICT 312, pp. 307-318, 2009.

(C) IFIP International Federation for Information Processing 2009 
In the present paper we determine numerically parameters in a Tikhonov regularization. This regularization technique is used to formulate the identification problem in terms of an optimal control problem. For any admissible parameter $p \in P_{\mathrm{ad}} \subset \mathbb{R}^{N}$ let $u(p)$ denote the solution to the underlying semilinear elliptic PDE. The identification problem is to find a parameter $p^{*} \in P_{\text {ad }}$ so that for a given (measurement) data $u_{d}$ (e.g., on the boundary or on a part of the domain) the quantity $\left\|u^{*}-u_{d}\right\|$ is minimal, where $u^{*}=u^{*}\left(p^{*}\right)$. For a precise introduction we refer to Sect. 2] For the Tikhonov regularization we take a $\kappa>0$ and solve the optimal control problem

$$
\min _{(p, u)} \frac{1}{2}\left\|u-u_{d}\right\|^{2}+\frac{\kappa}{2}\|p\|^{2} \quad \text { subject to (s.t.) } \quad u \text { solves PDE for } p \in P_{\mathrm{ad}} \text {. }
$$

By $\left(u^{\kappa}, p^{\kappa}\right)$ we denote a (local) optimal solution to (1). Then we introduce the following bilevel optimization problem:

$$
\min _{\kappa}\left\|u^{\kappa}-u_{d}\right\|^{2} \quad \text { s.t. } \quad\left(p^{\kappa}, u^{\kappa}\right) \text { solves (1) for } \kappa \geq \kappa_{a}
$$

with $\kappa_{a}>0$. To solve (2) numerically we apply the MATLAB routine fmincon, where the solution pair $\left(p^{\kappa}, u^{\kappa}\right)$ to (1) is computed by a fast optimization solver based on a POD Galerkin projection.

Note that the inner optimization problem (1) is non-convex, thus there might exist more than one local minimum. By varying the Tikhonov parameter $\kappa$ we search an optimal $\kappa^{*}$ so that (1) for $\kappa=\kappa^{*}$ yields a solution $\left(p^{*}, u^{*}\right)$ for which the error in a given norm between the state $u^{*}$ and the noisy measuring data $u_{d}$ is minimal.

A similar approach compared to the method of solving the bilevel problem above is to fix $\kappa$, but start the inner optimization loop with varying starting values $\left(p^{0}, u^{0}\right)$. In this work we only deal with the previous case (bilevel problem with varying $\kappa$ ), though. In both methods we exploit the fact that - using the POD approximation - one optimization loop takes very little time. Thus, it is no matter of temporal cost to solve an optimization problem like (1) many times successively.

The paper is organized in the following manner. In Sect. 2 we introduce the underlying parameter estimation problem. The POD method is briefly reviewed in Sect. 3 The POD basis is used to derive a POD Galerkin projection for the optimal control problem. Finally, numerical examples are carried out in the last section. In particular, we apply the reduced-basis method to obtain appropriate snapshots for the POD basis computation in one of the numerical tests.

\section{The Identification Problem}

Let $\Omega \subset \mathbb{R}^{d}, d=2,3$, be an open, bounded and connected set with Lipschitz-continuous boundary $\Gamma=\partial \Omega$. Let $q>d / 2+1$ and $r>d+1$. For given $f \in L^{q}(\Omega), g \in L^{r}(\Gamma)$, $c, q \in L^{\infty}(\Omega)$ with $c \geq c_{a}>0$ in $\Omega$ almost everywhere (a.e.) and $q \geq q_{a} \geq 0$ in $\Omega$ a.e., $\sigma \geq 0$ we consider the nonlinear problem

$$
\begin{aligned}
-c \Delta u+q u+e^{u}=f & \text { in } \Omega, \\
c \frac{\partial u}{\partial n}+\sigma u=g & \text { in } \Gamma .
\end{aligned}
$$


There exists a unique weak solution $u \in H_{b}^{1}(\Omega)=H^{1}(\Omega) \cap L^{\infty}(\Omega)$ satisfying

$$
\int_{\Omega} c \nabla u \cdot \nabla \varphi+\left(q u+e^{u}\right) \varphi \mathrm{d} \mathbf{x}+\int_{\Gamma} \sigma u \varphi \mathrm{d} \mathbf{s}=\int_{\Omega} f \varphi \mathrm{d} \mathbf{x}+\int_{\Gamma} g \varphi \mathrm{d} \mathbf{s}
$$

for all $\varphi \in H^{1}(\Omega)$, where the Banach space $H_{b}^{1}(\Omega)$ is endowed with the common norm $\|u\|_{H_{b}^{1}(\Omega)}=\|u\|_{H^{1}(\Omega)}+\|u\|_{L^{\infty}(\Omega)}$ for $u \in H_{b}^{1}(\Omega)$. Moreover, this solution belongs to $C(\bar{\Omega})$. For a proof we refer the reader to [4], for instance.

\subsection{Estimation of Diffusion and Potential Parameter}

The goal of the first estimation problem is to identify the parameter pair

$$
p=(c, q) \in P_{\mathrm{ad}}^{1}=\left\{\tilde{p}=(\tilde{c}, \tilde{q}) \in \mathbb{R}^{2} \mid \tilde{c} \geq c_{a} \text { and } \tilde{q} \geq q_{a}\right\}
$$

from measurements for the weak solution $u \in H_{b}^{1}(\Omega)$ to (3) on the boundary $\Gamma$ and on a subset $\Omega_{m}$ of the domain $\Omega$. Let $\alpha_{1}, \alpha_{2}$ denote nonnegative weights, $\kappa_{c}, \kappa_{q}$ be positive regularization parameters and $c_{d}, q_{d} \in \mathbb{R}$ stand for nominal parameters. Introducing the quadratic cost functional

$$
J_{1}(p, u)=\frac{\alpha_{1}}{2} \int_{\Gamma}\left|u-u_{\Gamma}\right|^{2} \mathrm{~d} \mathbf{s}+\frac{\alpha_{2}}{2} \int_{\Omega_{m}}\left|u-u_{\Omega}\right|^{2} \mathrm{~d} \mathbf{x}+\frac{\kappa_{c}}{2}\left|c-c_{d}\right|^{2}+\frac{\kappa_{q}}{2}\left|q-q_{d}\right|^{2}
$$

for $p=(c, q) \in \mathbb{R}^{2}$ and $u \in H^{1}(\Omega)$ we express the identification problem as the following constrained optimal control problem

$$
\min J_{1}(p, u) \quad \text { s.t. } \quad p=(c, q) \in P_{\text {ad }}^{1} \text { and } u \in H_{b}^{1}(\Omega) \text { satisfy (4). }
$$

Throughout the paper we suppose that (5) admits at least one local solution $x^{*}=\left(p^{*}, u^{*}\right)$ with $p^{*}=\left(c^{*}, q^{*}\right) \in P_{\mathrm{ad}}^{1}$.

\subsection{Estimation of Varying Diffusion Parameter}

In the second example we suppose that $\Omega$ is split into two measurable disjunct subsets $\Omega_{i}, i=1,2$, and that $c$ is constant on $\Omega_{i}$, i.e., $c \equiv c_{i}$ on $\Omega_{i}$ for $i=1,2$. Hence, we introduce the set of admissible parameters by

$$
P_{\mathrm{ad}}^{2}=\left\{\tilde{p}=\left(\tilde{c}_{1}, \tilde{c}_{2}\right) \in \mathbb{R}^{2} \mid \tilde{c}_{i} \geq c_{a} \text { for } i=1,2\right\} .
$$

The goal is to identify $c$ from given measurements for the weak solution $u \in H_{b}^{1}(\Omega)$ to (3) on the boundary $\Gamma$. Let $\alpha_{1}$ denote a nonnegative weight, $\kappa_{1}, \kappa_{2}$ be positive regularization parameters and $c_{1, d}, c_{2, d} \in \mathbb{R}$ stand for nominal potential parameters. Introducing the cost functional

$$
J_{2}(p, u)=\frac{\alpha_{1}}{2} \int_{\Gamma}\left|u-u_{\Gamma}\right|^{2} \mathrm{~d} \mathbf{s}+\frac{\kappa_{1}}{2}\left|c_{1}-c_{1, d}\right|^{2}+\frac{\kappa_{2}}{2}\left|c-c_{2, d}\right|^{2}
$$

for $p=\left(c_{1}, c_{2}\right) \in \mathbb{R}^{2}$ and $u \in H^{1}(\Omega)$ we express the identification problem as the following constrained optimal control problem

$$
\min J_{2}(p, u) \quad \text { s.t. } \quad p=\left(c_{1}, c_{2}\right) \in P_{\mathrm{ad}}^{2} \text { and } u \in H_{b}^{1}(\Omega) \text { satisfies (4). }
$$

We assume that (8) admits at least one local solution $x^{*}=\left(p^{*}, u^{*}\right)$ with $p^{*}=\left(c_{1}^{*}, c_{2}^{*}\right)$. 


\section{The POD Method}

In this section we introduce briefly the POD method. Suppose that for points $p_{j} \in P_{a d}^{i}$, $j=1, \ldots, n$ and $i=1,2$, we know (at least approximately) the solution $u_{j}$ to (3), e.g., by utilizing a finite element or finite difference discretization. We set

$$
\mathcal{V}=\operatorname{span}\left\{u_{1}, \ldots, u_{n}\right\} \subset H_{b}^{1}(\Omega) \subset H^{1}(\Omega)
$$

with $d=\operatorname{dim} \mathcal{V} \leq n$. Then the POD basis of rank $\ell \leq d$ is given by the solution to

$$
\min _{\psi_{1}, \ldots, \psi_{\ell}} \sum_{j=1}^{n} \beta_{j}\left\|u_{j}-\sum_{i=1}^{\ell}\left\langle u_{j}, \psi_{i}\right\rangle_{H^{1}(\Omega)} \psi_{i}\right\|_{H^{1}(\Omega)}^{2} \quad \text { s.t. } \quad\left\langle\psi_{i}, \psi_{j}\right\rangle_{H^{1}(\Omega)}=\delta_{i j}
$$

with nonnegative weights $\left\{\beta_{j}\right\}_{j=1}^{n}$. For the choice of the $\beta_{j}$ 's we refer to [11]14].

The solution to (10) is characterized by the eigenvalue problem

$$
\mathscr{R} \psi_{i}=\lambda_{i} \psi_{i}, \quad 1 \leq i \leq \ell,
$$

where $\lambda_{1} \geq \lambda_{2} \geq \ldots \geq \lambda_{\ell} \geq \ldots \geq \lambda_{d}>0$ denote the eigenvalues of the linear, bounded, self-adjoint, and nonnegative operator $\mathscr{R}: H^{1}(\Omega) \rightarrow \mathcal{V}$ defined by

$$
\mathscr{R} z=\sum_{j=1}^{n} \beta_{j}\left\langle u_{j}, z\right\rangle_{H^{1}(\Omega)} u_{j} \quad \text { for } z \in H^{1}(\Omega) ;
$$

see [7]14[21]. Suppose that we have determined a POD basis $\left\{\psi_{i}\right\}_{i=1}^{\ell}$. We set

$$
V^{\ell}=\operatorname{span}\left\{\psi_{1}, \ldots, \psi_{\ell}\right\} \subset \mathcal{V} \subset H^{1}(\Omega) .
$$

Then the following relation holds

$$
\sum_{j=1}^{n} \beta_{j}\left\|u_{j}-\sum_{i=1}^{\ell}\left\langle u_{j}, \psi_{i}\right\rangle_{H^{1}(\Omega)} \psi_{i}\right\|_{H^{1}(\Omega)}^{2}=\sum_{i=\ell+1}^{d} \lambda_{i}
$$

i.e., a rapid decay of the eigenvalues $\lambda_{i}$ indicates that the vectors $u_{1}, \ldots, u_{n}$ can be well approximated by taking only a few ansatz functions $\left\{\psi_{i}\right\}_{i=1}^{\ell}$ with $\ell \ll d$.

Now we introduce the POD Galerkin scheme for (4) as follows: the function $u^{\ell}=$ $\sum_{i=1}^{\ell} u_{i}^{\ell} \psi_{i} \in V^{\ell}$ solves

$$
\begin{array}{r}
\int_{\Omega} c \nabla u^{\ell} \cdot \nabla \psi \mathrm{d} \mathbf{x}+\int_{\Omega}\left(q u^{\ell}+e^{u^{\ell}}\right) \psi \mathrm{d} \mathbf{x}+\int_{\Gamma} \sigma u^{\ell} \psi \mathrm{d} \mathbf{s} \\
=\int_{\Omega} f \psi \mathrm{d} \mathbf{x}+\int_{\Gamma} g \psi \mathrm{d} \mathbf{s} \quad \text { for all } \psi \in V^{\ell} .
\end{array}
$$

Problem (15) is a nonlinear system for the $\ell$ unknown modal coefficients $u_{1}^{\ell}, \ldots, u_{\ell}^{\ell} \in \mathbb{R}$. If

$$
\mathscr{E}(\ell)=\frac{\sum_{i=1}^{\ell} \lambda_{i}}{\sum_{i=1}^{d} \lambda_{i}} \approx 1 \text { for } \ell \ll d,
$$

holds, (15) is called a low-dimensional model for (4). 


\section{Numerical Experiments}

In this section we present numerical examples for the identification problem. The numerical tests are executed on a standard 3.0 GHz desktop PC. We are using the MATLAB 7.1 package together with FEMLAB 3.1.

Run 1 (Problem (5)). Suppose that the domain $\Omega$ is given by

$$
\Omega=\left\{\mathbf{x}=\left(x_{1}, x_{2}\right) \mid \frac{x_{1}^{2}}{1.2^{2}}+x_{2}^{2}<1\right\} \subset \mathbb{R}^{2} ;
$$

see Fig. 11 In (3) we choose $f=5, \sigma=3 / 2$, and $g=-1$. For $c_{e x}=1.2$ and $q_{e x}=11$ we calculate a finite element (FE) solution $u_{e x}^{h}=u^{h}\left(c_{e x}, q_{e x}\right)$ with 1275 degrees of freedom. The parameter $p_{e x}=\left(c_{e x}, q_{e x}\right)$ is our reference parameter.

$$
\Omega, \Omega_{\mathrm{m}} \text {, and points where data is available }
$$

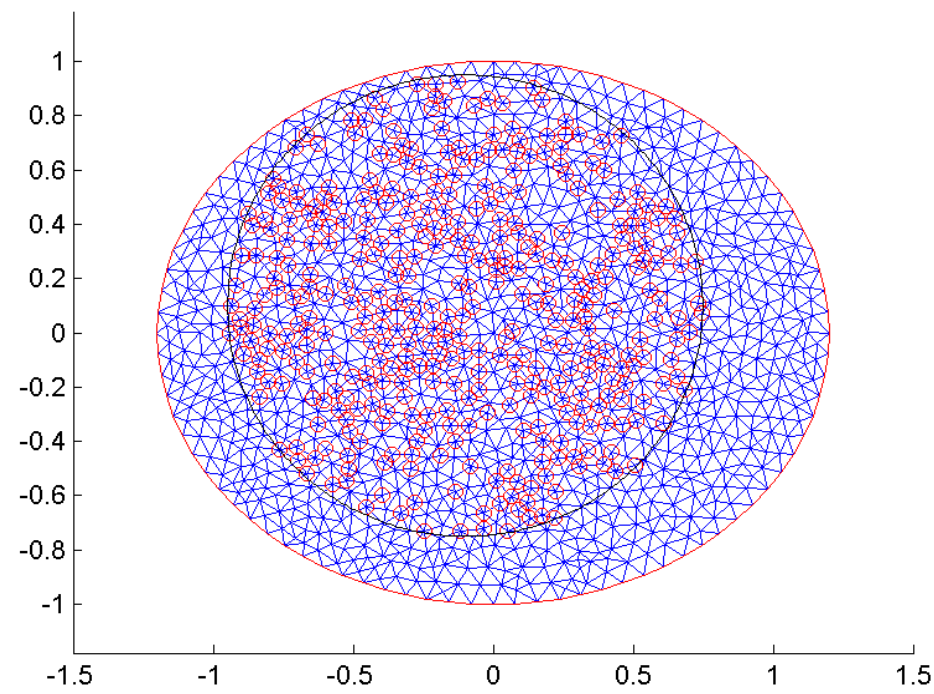

Fig. 1. Run 1 Domain $\Omega$ and the interior points for which we have measurements

Basis computation. We distinguish three different techniques in deriving a basis for the Galerkin projection.

1) First we compute 20 snapshots by varying the parameters $c$ and $q$ simultaneously. We define the equidistant grid

$$
(c, q) \in\{0.2,0.8,1.4,2\} \times\{1,8,15,22,29\}
$$


and calculate a POD model with $\ell=6$ basis functions. In 10 we choose trapezoidal weights. Thus, we consider

$$
\min \sum_{i=1}^{4} \sum_{j=1}^{5} \beta_{i} \tilde{\beta}_{j}\left\|u^{h}\left(c_{i}, q_{j}\right)-\sum_{k=1}^{\ell}\left\langle u^{h}\left(c_{i}, q_{j}\right), \psi_{k}\right\rangle_{H^{1}(\Omega)} \psi_{k}\right\|_{H^{1}(\Omega)}^{2}
$$

where

$$
\beta_{1}=\frac{c_{2}-c_{1}}{2}, \quad \beta_{i}=\frac{c_{i+1}-c_{i-1}}{2} \text { for } i=2,3, \quad \beta_{4}=\frac{c_{4}-c_{3}}{2}
$$

and

$$
\tilde{\beta}_{1}=\frac{q_{2}-q_{1}}{2}, \quad \tilde{\beta}_{j}=\frac{q_{j+1}-q_{j-1}}{2} \text { for } j=2,3,4, \quad \tilde{\beta}_{5}=\frac{q_{5}-q_{4}}{2} .
$$

The relative error in the $H^{1}$-norm between the FE state $u_{e x}^{h}$ and the POD state $u_{e x}^{\ell}=$ $u^{\ell}\left(c_{e x}, q_{e x}\right)$ is $6.2 \cdot 10^{-4}$.

2) Alternatively, we use the reduced-basis method (see [8]16]18], for instance) in order to obtain a 6-dimensional model of the elliptic system. The idea of the reducedbasis method is to choose the parameter instances for which the snapshots are computed intelligently and to use these snapshots directly as basis in the Galerkin projection. Therefore we apply the simplified formula taken from [17]:

$$
q_{k}^{r b}=\exp \left(-\ln \gamma+k \cdot \delta^{q}\right)-\frac{1}{\gamma} \quad \text { for } k=1, \ldots, N
$$

where we set $\gamma=0.02, q_{\max }=29, N=3$, and $\delta^{q}=\ln \left(\gamma \cdot q_{\max }+1\right) / N$. Hence, we find that the parameters for which the snapshots should be computed are: $q_{1}^{r b}=4.3$, $q_{2}^{r b}=12.68$, and $q_{3}^{r b}=29$. Analogously we set $\gamma=0.02, c_{\max }=2, M=2$, and $\delta^{c}=\ln \left(\gamma \cdot c_{\text {max }}+1\right) / M$ and choose

$$
c_{k}^{r b}=\exp \left(-\ln \gamma+k \cdot \delta^{c}\right)-\frac{1}{\gamma}, k=1, \ldots, M,
$$

hence we find $c_{1}^{r b}=0.91$ and $c_{2}^{r b}=2$. Thus, the 6 reduced-basis elements are the solutions $u^{h}(c, q)$ to (3) computed for the parameter instances

$$
(c, q) \in\{0.91,2\} \times\{4.3,12.68,29\}
$$

The relative error in the $H^{1}$-norm between the FE state $u_{e x}^{h}$ and the reduced order model $u_{e x}^{r b}=u^{r b}\left(c_{e x}, q_{e x}\right)$ is $1.7 \cdot 10^{-4}$.

3) The best approximation of the FE state can be obtained by combining both methods (POD and reduced-basis). Therefore we compute 20 snapshots at the parameter instances calculated by the reduced-basis ansatz (i.e., we set $N=5$ and $M=4$ and use the formula from above again). We find that the snapshots should be computed at the 20 snapshot pairings

$$
(c, q) \in\{0.43,0.91,1.43,2\} \times\{2.23,5.57,10.53,17.95,29\} .
$$


Then we construct a 6-dimensional POD basis. The relative error in the $H^{1}$-norm between the FE state $u_{e x}^{h}$ and this reduced order model $u_{e x}^{\ell, r b}=u^{\ell, r b}\left(c_{e x}, q_{e x}\right)$ is now about $10^{-4}$.

We proceed by using this POD basis for the reduced-order modeling. The computation of the POD solution takes 437 seconds (411 seconds thereof are for the computation of the $20 \mathrm{FE}$ snapshots whereas one solve of the nonlinear POD model only takes 0.06 seconds). From Table 1 it can be observed that the relative error between the FE state and the POD state decreases as the number of POD basis functions increases.

Table 1. Run 1 Relative errors between the FE state and the POD state for increasing number of POD basis functions

\begin{tabular}{rlll}
\hline & $\ell=4 \quad \ell=5 \quad \ell=6 \quad \ell=7$ \\
\hline$\frac{\left\|u^{h}-u^{\ell, r b}\right\|_{H^{1}(\Omega)}}{\left\|u^{h}\right\|_{H^{1}(\Omega)}} 1.2 \mathrm{e}-3$ & $5.3 \mathrm{e}-4$ & $1.0 \mathrm{e}-4$ & $1.1 \mathrm{e}-5$ \\
\hline
\end{tabular}

Identification problem. Now turn to the identification problem. Let $c_{a}=q_{a}=0.01$ to ensure that both parameters are positive. Moreover, we choose $c_{d}=q_{d}=0$, i.e., no apriori knowledge on the parameters is available. We add a random noise of $8 \%$ to the FE state $u_{e x}^{h}$. For the weights in the cost functional we take $\alpha_{1}=\alpha_{2}=1000$, and we choose

$$
\Omega_{m}=\left\{\mathbf{x}=\left(x_{1}, x_{2}\right) \in \Omega \mid\left(x_{1}+0.1\right)^{2}+\left(x_{2}-0.1\right)^{2}<0.85^{2}\right\}
$$

for the partial measurement. Furthermore, we suppose that measurements are not given on the whole subdomain $\Omega_{m}$, but only on 381 points (of totally 762 grid points) in $\Omega_{m}$. The points for which we have measurements (besides the points on the boundary) are indicated by the circles in Fig. 1 Now we consider the bilevel optimization problem (compare (2))

$$
\min _{\kappa=\left(\kappa_{c}, \kappa_{q}\right)} \int_{\Gamma}\left|u^{\kappa}-u_{\Gamma}\right|^{2} \text { ds } \quad \text { s.t. } \quad\left(c^{\kappa}, q^{\kappa}, u^{\kappa}\right) \text { solves (5) for } \kappa_{c}, \kappa_{q} \geq 10^{-16}
$$

By using the MATLAB function fmincon we determine - after 56.2 seconds - the optimal weighting parameters $\kappa_{c}^{*}=0.1691$ and $\kappa_{q}^{*}=10^{-16}$. For these optimal weights we solve the reduced order model by means of an augmented Lagrange-SQP algorithm and use the POD Galerkin projection. Altogether 50 SQP iterations are required and we find numerically an optimal solution $\left(c^{*}, q^{*}, u^{*}\right)$ to 27]; in particular, $c^{*}=1.1972$ and $q^{*}=10.9827$. Thus,

$$
\frac{\left\|p_{e x}-p^{*}\right\|_{2}}{\left\|p_{e x}\right\|_{2}} \approx 0.16 \% \text { with } p_{e x}=\left(c_{e x}, q_{e x}\right) \text { and } p^{*}=\left(c^{*}, q^{*}\right) .
$$

The relative errors in the state variable to the exact (unnoisy) data and to the noisy data are stated for 3 different norms in Table 2 The CPU time for the optimization is small compared to the POD computation time. The POD optimization algorithm for (5) 
Table 2. Run 11 Relative errors of the suboptimal state $u^{*}$ compared to the exact data $u_{e x}^{h}$ and to the noisy data $u_{\Gamma}$ for the optimal $\left(\kappa_{c}^{*}, \kappa_{q}^{*}\right)=\left(0.1691,10^{-16}\right)$ and for $\left(\kappa_{c}^{(j)}, \kappa_{q}^{(j)}\right), j=1,2,3$

\begin{tabular}{lcccc}
\hline & \multicolumn{1}{c}{$\frac{\left\|u^{*}-u\right\|_{L^{2}(\Gamma)}}{\|u\|_{L^{2}(\Gamma)}} \frac{\left\|u^{(1)}-u\right\|_{L^{2}(\Gamma)}}{\|u\|_{L^{2}(\Gamma)}}$} & $\frac{\left\|u^{(2)}-u\right\|_{L^{2}(\Gamma)}}{\|u\|_{L^{2}(\Gamma)}}$ & $\frac{\left\|u^{(3)}-u\right\|_{L^{2}(\Gamma)}}{\|u\|_{L^{2}(\Gamma)}}$ \\
\hline$u=u_{e x}^{h}$ & 0.004592 & 0.091625 & 0.013806 & 0.018451 \\
$u=u_{\Gamma}$ & 0.037749 & 0.095162 & 0.042008 & 0.044252 \\
\hline
\end{tabular}

only takes 1.7 seconds. For comparison, when we use the FE discretized model in the augmented SQP-Lagrange algorithm, it takes about 290 seconds to obtain a solution. Note that for the choice $\kappa_{c}^{(1)}=5 \cdot \kappa_{c}^{*}$ and $\kappa_{q}^{(1)}=\kappa_{q}^{*}$, we find the solution $c^{(1)}=1.1746$ and $q^{(1)}=10.9273$, which gives

$$
\frac{\left\|p_{e x}-p^{(1)}\right\|_{2}}{\left\|p_{e x}\right\|_{2}} \approx 0.7 \% \quad \text { with } p^{(1)}=\left(c^{(1)}, q^{(1)}\right)
$$

and the relative errors are as stated in Table 2. The same can be done with $\kappa_{c}^{(2)}=0.2 \cdot \kappa_{c}^{*}$ and $\kappa_{q}^{(2)}=\kappa_{q}^{*}$. We find $c^{(2)}=1.2021$ and $q^{(2)}=10.9947$. Thus,

$$
\frac{\left\|p_{e x}-p^{(2)}\right\|_{2}}{\left\|p_{e x}\right\|_{2}} \approx 0.05 \% \quad \text { with } p^{(2)}=\left(c^{(2)}, q^{(2)}\right) .
$$

Finally, we choose $\kappa_{c}^{(3)}=\kappa_{q}^{(3)}=10^{-16}$. The resulting parameters are $c^{(3)}=1.2034$ and $q^{(3)}=10.9978$, which gives

$$
\frac{\left\|p_{e x}-p^{(3)}\right\|_{2}}{\left\|p_{e x}\right\|_{2}} \approx 0.04 \% \quad \text { with } p^{(3)}=\left(c^{(3)}, q^{(3)}\right)
$$

We observe that the relative error in the coefficients is smaller for both $p^{(2)}$ and $p^{(3)}$ compared to $p^{*}$. However, we observe from Table 2 that the relative errors of the PDE solution $u^{*}$ on the boundary $\Gamma$ are the smallest ones. Note that in (27) the term $\left\|u-u_{\Gamma}\right\|^{2}$ is minimized. For the absolute errors we refer to Table 3 . Also the absolute errors are for $\kappa^{*}$ the smallest ones, in particular also the error of $u^{*}-u_{e x}^{h}$.

\begin{tabular}{|c|c|c|c|c|}
\hline \multicolumn{5}{|c|}{$\left\|u^{*}-u\right\|_{L^{2}(\Gamma)}\left\|u^{(1)}-u\right\|_{L^{2}(\Gamma)}\left\|u^{(2)}-u\right\|_{L^{2}(\Gamma)}\left\|u^{(3)}-u\right\|_{L^{2}(\Gamma}$} \\
\hline$u=u_{e x}^{h}$ & 0.000166 & 0.00 & 0.000500 & 0.00 \\
\hline$u=u_{\Gamma}$ & 0.001363 & 0.003437 & 0.001517 & 0.001598 \\
\hline
\end{tabular}

Table 3. Run 11 Absolute errors of the suboptimal state $u^{*}$ compared to the exact data $u_{e x}^{h}$ and to the noisy data $u_{\Gamma}$ for the optimal $\left(\kappa_{c}^{*}, \kappa_{q}^{*}\right)=\left(0.1691,10^{-16}\right)$ and for $\left(\kappa_{c}^{(j)}, \kappa_{q}^{(j)}\right), j=1,2,3$ 


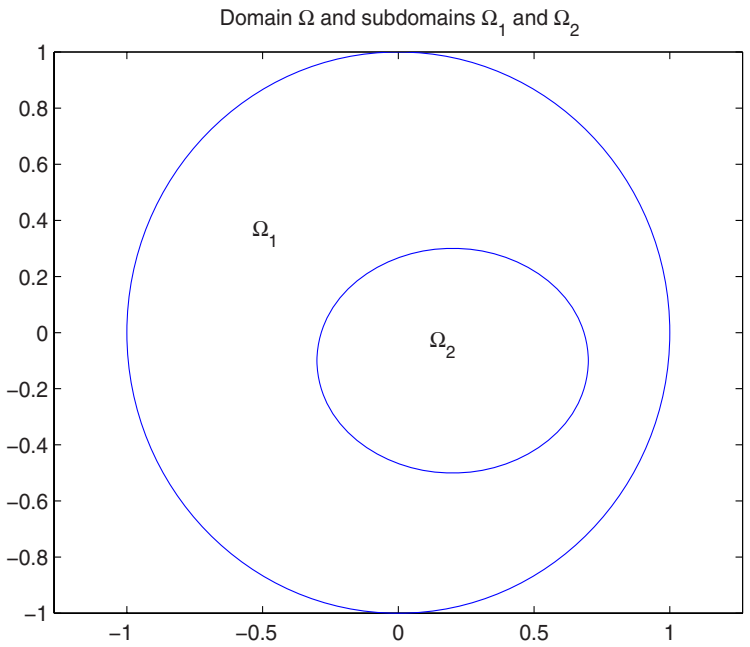

Fig. 2. Run 2 Domain $\Omega$ and subdomains $\Omega_{1}, \Omega_{2}$

Run 2 (Problem (8)). Now let $\Omega=\left\{\mathbf{x}=\left(x_{1}, x_{2}\right) \mid x_{1}^{2}+x_{2}^{2}<1\right\}$ be the open unit circle in $\mathbb{R}^{2}$ and the subdomains $\Omega_{1}, \Omega_{2}$ be given as

$$
\Omega_{1}=\Omega \backslash \Omega_{2}, \quad \Omega_{2}=\left\{\mathbf{x}=\left(x_{1}, x_{2}\right) \in \Omega \mid \frac{\left(x_{1}-0.2\right)^{2}}{a^{2}}+\frac{\left(x_{2}+0.1\right)^{2}}{b^{2}}<1\right\}
$$

with $a=0.5$ and $b=0.4$; see Fig. 2. In (3) we choose $q \equiv 20, f \equiv 4, \sigma=2$, and $g(\mathbf{x})=10+\cos \left(\pi x_{1} / 2\right) \cdot \cos \left(\pi x_{2} / 2\right)$. For $p_{e x}=\left(c_{1, e x}, c_{2, e x}\right)=(0.8,1.3)$ we compute the FE solution with 1070 degrees of freedom. To derive a POD basis we choose the diffusion values $p_{j}=\left(\eta_{k}, \eta_{l}\right) \in \mathbb{R}_{+}^{2}, 1 \leq j \leq n$, with

$$
j=5(k-1)+l \text { for } 1 \leq k, l \leq 5, \quad \eta_{k}=0.5+\frac{k-1}{4} \text { for } k=1, \ldots, 5
$$

and compute the corresponding FE solutions $u_{j}^{h}=u^{h}\left(p_{j}\right) \in H^{1}(\Omega)$ to (3), i.e., we have $n=25$ snapshots $\left\{u_{j}^{h}\right\}_{j=1}^{n}$. The computation of the snapshots requires 307 seconds. Next we compute the POD basis of rank $\ell=7$ as described in Sect. 3 and construct the POD model $u^{\ell}(\bar{c})$ which has a relative error to the FE state $u_{e x}^{h}$ of $1.38 \cdot 10^{-4}$. Now (2) has the form

$$
\min _{\kappa=\left(\kappa_{1}, \kappa_{2}\right)} \int_{\Gamma}\left|u^{\kappa}-u_{\Gamma}\right|^{2} \mathrm{ds} \quad \text { s.t. } \quad\left(c_{1}^{\kappa}, c_{2}^{\kappa}, u^{\kappa}\right) \text { solves (8) for } \kappa_{1}, \kappa_{2} \geq 10^{-16} .
$$

In the optimization algorithm for noisy data (3\%) we choose $\alpha_{1}=100$ and find the optimal weight $\kappa^{*}=\left(\kappa_{1}^{*}, \kappa_{2}^{*}\right)=(0.7534,0.0023)$. The corresponding optimal coefficient is $p^{*}=(0.7873,1.3247)$. Moreover, the relative and absolute errors in the state variable are stated in Table 4 If we take $\kappa^{(1)}=\left(\kappa_{1}^{(1)}, \kappa_{2}^{(1)}\right)=\left(10^{-16}, 10^{-16}\right)$ instead of $\kappa^{*}$, 
Table 4. Run 2 Relative errors of the suboptimal state $u^{*}$ compared to the exact data $u_{e x}^{h}$ and to the noisy data $u_{\Gamma}$ for $\kappa_{1}=0.7534$ and $\kappa_{2}=0.0023$

\begin{tabular}{lcc}
\hline & $\frac{\left\|u^{*}-u\right\|_{L^{2}(\Gamma)}}{\|u\|_{L^{2}(\Gamma)}}\left\|u^{*}-u\right\|_{L^{2}(\Gamma)}$ \\
\hline$u=u_{e x}^{h}$ & 0.004276 & 0.016811 \\
$u=u_{\Gamma}$ & 0.012713 & 0.050184 \\
\hline
\end{tabular}

the result is $p^{(1)}=(0.7902,1.4185)$ solves $(8)$. Then, $\left\|p_{\text {ex }}-p^{(1)}\right\|_{2} /\left\|p_{\text {ex }}\right\|_{2} \approx 8 \%$, but $\left\|p_{\text {ex }}-p^{*}\right\|_{2} /\left\|p_{\text {ex }}\right\|_{2} \approx 2 \%$.

Now, let the subdomains $\Omega_{1}$ and $\Omega_{2}$ be given as

$$
\Omega_{1}=\Omega \backslash \Omega_{2}, \quad \Omega_{2}=\left\{\mathbf{x}=\left(x_{1}, x_{2}\right) \in \Omega \mid x_{1}^{2}+\left(x_{2}+0.1\right)^{2}<0.75^{2}\right\} .
$$

We choose $p_{e x}=\left(c_{1, e x}, c_{2, e x}\right)=(1.2,0.9)$, all other parameters in (3) remain the same. Moreover, the measuring data $u_{d}$ is much more noisy (15\%) than before. In this case we observe that - due to the bigger noise - both components of the ideal $\kappa^{*}$ are far away from zero (see Fig. 3). The cost funtional in (2) for $\kappa^{(1)}=\left(10^{-16}, 10^{-16}\right)$ has a value of 0.2757 , while for $\kappa^{*}=(0.3465,0.6675)$ the cost is only 0.2745 . However, the relative error in the parameter $p=\left(p_{1}, p_{2}\right)$ is much smaller for the solution using $\kappa^{(1)}$ rather than $\kappa^{*}$. We observe $\left\|p_{e x}-p^{(1)}\right\|_{2} /\left\|p_{e x}\right\|_{2} \approx 0.8 \%$, but $\left\|p_{e x}-p^{*}\right\|_{2} /\left\|p_{e x}\right\|_{2} \approx 14 \%$.
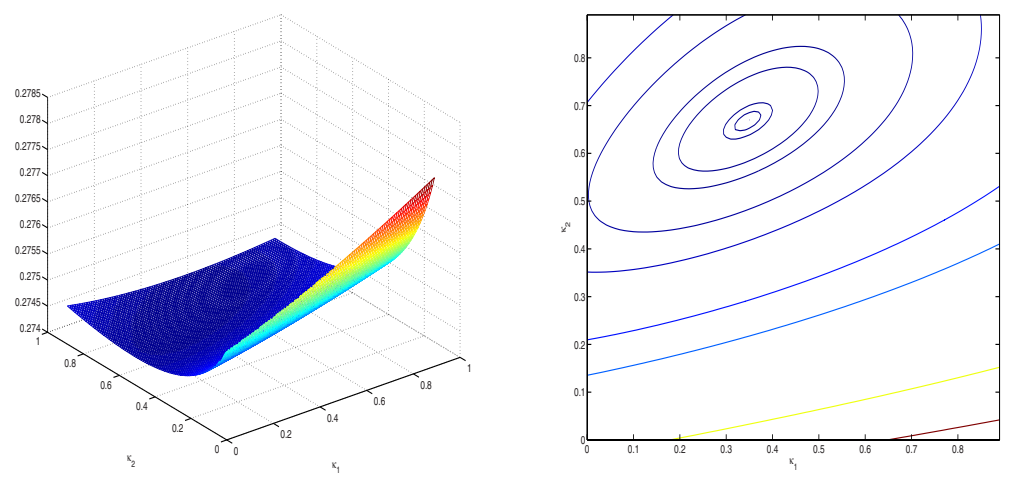

Fig. 3. Run2. Cost functional in (2) for a grid of different $\kappa=\left(\kappa_{1}, \kappa_{2}\right)$ (left plot) and contour plot of the cost functional. The absolute minimum is approximately at $\kappa^{*}=(0.35,0.67)$ (right plot).

Acknowledgements. The authors gratefully acknowledge partial support by the Austrian Science Fund FWF under grant no. P19588-N18 and by the SFB Research Center "Mathematical Optimization in Biomedical Sciences" (SFB F32). 


\section{References}

1. Barrault, M., Maday, Y., Nguyen, N.C., Patera, A.T.: An empirical interpolation method: application to efficient reduced-basis discretization of partial differential equations. Comptes Rendus de'l Académie des Sciences Paris I(339), 667-672 (2004)

2. Bertsekas, D.P.: Constrained Optimization and Lagrange Multipliers. Academic Press, New York (1982)

3. Bertsekas, D.P.: Nonlinear Programming, 2nd edn. Athena Scientific, Belmont (1999)

4. Casas, E., Raymond, J.-P., Zidani, H.: Optimal control problem governed by semilinear elliptic equations with integral control constraints and pointwise state constraint. In: Desch, W., et al. (eds.) Control and estimation of distributed parameter systems. International conference in Vorau, Austria, July 14-20. Birkhauser, Basel (1996); ISNM, Int. Ser. Numer. Math. 126, 89-102 (1998)

5. Fukuda, K.: Introduction to Statistical Recognition. Academic Press, New York (1990)

6. Hintermüller, M.: A primal-dual active set algorithm for bilaterally control constrainted optimal control problems. Quarterly of Applied Mathematics 61, 131-160 (2003)

7. Holmes, P., Lumley, J.L., Berkooz, G.: Turbulence, Coherent Structures, Dynamical Systems and Symmetry. In: Cambridge Monographs on Mechanics. Cambridge University Press, Cambridge (1996)

8. Ito, K., Ravindran, S.S.: Reduced basis method for unsteady viscous flows. Int. J. of Comp. Fluid Dynamics 15, 97-113 (2001)

9. Lall, S., Marsden, J.E., Glavaski, S.: Empirical model reduction of controlled nonlinear systems. In: Proceedings of the IFAC Congress, vol. F, pp. 473-478 (1999)

10. Ly, H.V., Tran, H.T.: Modelling and control of physical processes using proper orthogonal decomposition. Mathematical and Computer Modeling 33, 223-236 (2001)

11. Kahlbacher, M., Volkwein, S.: Galerkin proper orthogonal decomposition methods for parameter dependent elliptic systems. Discussiones Mathematicae: Differential Inclusions, Control and Optimization 27, 95-117 (2007)

12. Kahlbacher, M., Volkwein, S.: Model reduction by proper orthogonal decomposition for estimation of scalar parameters in elliptic PDEs. In: Wesseling, P., Onate, E., Periaux, J. (eds.) Proceedings of ECCOMAS CFD, Egmont aan Zee (2006)

13. Kahlbacher, M., Volkwein, S.: Estimation of diffusion coefficients in a scalar GinzburgLandau equation by using model reduction. Submitted (2007),

http://www. uni-graz.at/imawww/reports/archive-2007/IMA05-07. pdf

14. Kunisch, K., Volkwein, S.: Galerkin proper orthogonal decomposition methods for a general equation in fluid dynamics. SIAM J. Numer. Anal. 40, 492-515 (2002)

15. Sirovich, L.: Turbulence and the dynamics of coherent structures, parts I-III. Quarterly of Applied Mathematics XLV, 561-590 (1987)

16. Machiels, L., Maday, Y., Patera, A.T.: Output bounds for reduced-order approximations of elliptic partial differential equations. Computer Methods in Applied Mechanics and Engineering 190, 3413-3426 (2001)

17. Maday, Y., Patera, A.T., Turinici, G.: Global a priori convergence theory for reduced-basis approximations of single-parameter symmetric coercive elliptic partial differential equations. Comptes Rendus de'l Académie des Sciences Paris I(335), 289-294 (2002)

18. Maday, Y., Rønquist, E.M.: A reduced-basis element method. Journal of Scientific Computing 17, 1-4 (2002)

19. Rowley, C.W.: Model reduction for fluids, using balanced proper orthogonal decomposition. International Journal of Bifurcation and Chaos 15, 997-1013 (2005) 
20. Vogel, C.R.: Computational Methods for Inverse Problems, Philadlphia. SIAM Frontiers in Applied Mathematics (2002)

21. Volkwein, S.: Model Reduction using Proper Orthogonal Decomposition. Lecture Notes, Institute of Mathematics and Scientific Computing, University of Graz, http://www.uni-graz.at/imawww/volkwein/POD.pdf

22. Volkwein, S., Hepberger, A.: Impedance Identification by POD Model Reduction Techniques (2008) (submitted), http://www.uni-graz.at/imawww/reports/archive-2008/IMA01-08. pdf

23. Willcox, K., Peraire, J.: Balanced model reduction via the proper orthogonal decomposition. American Institute of Aeronautics and Astronautics (AIAA) 40, 2323-2330 (2002) 